\title{
Application of the solid state NMR to the study of the alcohol/alkane mixtures adsorption onto graphite
}

- María D. Alba ${ }^{a}$, , Miguel A. Castro ${ }^{a}$, Stuart M. Clarke ${ }^{b}$, Santiago Medinaa, Loic Messe ${ }^{b}$, Carmen Millán ${ }^{\mathrm{a}}$

- a Instituto Ciencia de los Materiales de Sevilla (CSIC-Universidad de Sevilla), Avda. Americo Vespucio 49, 41092 Sevilla, Spain

- ${ }^{\mathrm{b}}$ Department of Chemistry and BP Institute, University of Cambridge, Cambridge CB3 OEZ, United Kingdom

\begin{abstract}
The mixing of molecules adsorbed from solution to different interfaces has both industrial and academic relevance and the mixing behaviour at the interface is a key to understanding for example, that the surface tension of a mixture of two surfactants is lower than either of the two pure materials and many other effects. In this paper, we report, for the first time, the application of Solid State NMR to the study of alkane/alcohol mixtures, in a range of relative size ratio between 0 and 0.35 , adsorbed onto graphite at high, multilayer coverage. Moreover, this paper evaluated, for the first time, the utility of the combined used of ${ }^{1} \mathrm{H}$ and ${ }^{2} \mathrm{H}$ NMR for: (i) determining the surface composition and (ii) making a theoretical approach to the sorption isotherm. A variety of preferential adsorption behaviour is reported. Preferential adsorption of the longer molecule (decane vs. heptanol) from a mixture has been observed. However, if both components are of similar length, the alcohol is preferentially adsorbed (heptanol vs. octane and octanol vs. octane). Finally, a linear relation between the relative size ratio and the amount of alcohol at monolayer coverage is observed.
\end{abstract}

Keywords Solid State NMR; Graphite; Alkane; Alcohol; Adsorption; Monolayer

\section{Introduction}

The mixing of molecules adsorbed from solution to different interfaces has both industrial and academic relevance and the mixing behaviour at the interface is a key to understanding for example, that the surface tension of a mixture of two surfactants is lower than either of the two pure materials and many other effects [1]. Many studies have been performed to analyze the behaviour of solid monolayers adsorbed from solutions of binary mixtures of linear alcohols and binary mixtures of linear alkanes [1], [2], [3], [4] and [5]. Among them, a previous study of alkane/alcohol mixed monolayers at the solid/liquid interface at submonolayer coverage demonstrated that alcohols are preferentially adsorbed over alkanes [1]. For example, an alcohol of chain length $m$ can adsorb equally as strong as an alkane two methylene groups longer. It was also demonstrated that a relative size ratio (RS) of 0.2 will give approximately equal preferential adsorption between an alcohol and an alkane. However, no experimental evidence of the adsorption isotherm alkane/alcohol mixtures adsorbed onto graphite has been reported. 
A combination of several experimental techniques has been applied during the last decade to this study to provide detailed structural and compositional information on multi-component systems adsorbed on graphite. Among them, Incoherent elastic neutron scattering, IQNS, is employed for identifying and characterizing monolayers adsorbed from pure materials and solutions. This technique provides unambiguous information on both the state and absolute composition of an adsorbed layer as well as other information such as estimates of the area per molecule on the surface [6] and [7]. However, IQNS require centralized international facilities to produce the neutrons and the measurements are time-consuming, costly and difficult to carry out. Hence, there is great interest in developing complementary techniques that can survey the temperature and composition behaviour more readily. Nuclear magnetic resonance, NMR, has recently provided to be an efficient tool to determine the phase transitions of adsorbed species and has been satisfactorily applied to study the behaviour of $\mathrm{n}$ dodecane adsorbed on graphite and the behaviour of the decane-heptanol system [8] and [9]. In outline, the technique exploits the 'dynamic contrast' between the 'immobile' adsorbed species and the mobile species in the bulk liquid. Significantly the complementary information obtained from combining ${ }^{1} \mathrm{H}$ and ${ }^{2} \mathrm{H}$ NMR signals from both deuterated and protonated samples has indicated that this exciting methodology can be extended to the study of multicomponent systems and to obtain independent information on each component.

Although the decane-heptanol system was previously explored in Ref. [9], in this paper, we report, for the first time, the application of Solid State NMR to the study of an extended range of alkane/alcohol mixtures, in a range of relative size ratio between 0 and 0.35 , adsorbed onto graphite at high, multilayer coverage. Moreover, this paper evaluates, for first time, the utility of the combined used of ${ }^{1} \mathrm{H}$ and ${ }^{2} \mathrm{H}$ NMR for: (i) determining the surface composition and (ii) making a theoretical approach to the sorption isotherm.

\section{Experimental}

\subsection{Materials}

A powdered and graphitized carbon black Carbopack B (Supelco Inc., Bellefonte, USA) [10] with a specific surface area of $98.1 \mathrm{~m}^{2} \mathrm{~g}^{-1}$ was employed as adsorbent. Non-deuterated decane ( $\mathrm{h}$ decane) and octane (h-octane) and deuterated heptanol (d-heptanol) and octanol (d-octanol) were obtained from Aldrich and used without further purification.

Graphite substrates were outgassed under vacuum in an oven at $350{ }^{\circ} \mathrm{C}$ before known quantities of alcohol/alkane mixtures were added as liquid by microsyringe. The system was annealed onto the surface at a temperature of approximately $40{ }^{\circ} \mathrm{C}$ below the bulk boiling point. We express the amount of each adsorbed component in terms of the number of equivalent monolayers adsorbed. This is estimated from the areas per molecule, taken from Groszek [11] and Morishige [12], and the specific surface area of the graphite. Approximately 8 monolayers of deuterated alcohols were used and successive amounts of non-deuterated alkanes were added to achieve the different required compositions.

\subsection{Solid nuclear magnetic resonance}

Static ${ }^{1} \mathrm{H}$ and ${ }^{2} \mathrm{H}$ NMR measurements at $9.39 \mathrm{~T}$ were performed on a Bruker DRX400 spectrometer at NMR Research Service, Spectroscopics Service at ICMS (CSIC-University of Sevilla), equipped with a multinuclear probe and an analogical/digital ADC $2 \mathrm{MHz}$ converter. The spectra were recorded at different temperatures after an equilibration time of $5 \mathrm{~min}$. Longer equilibration times were tested during the acquisition in order to ensure that the 
selected time was long enough. ${ }^{1} \mathrm{H}$ and ${ }^{2} \mathrm{H}$ NMR spectra were obtained using single pulse sequence and a typical $\pi / 2$ pulse widths of $4.1 \mu \mathrm{s}$ and a pulse space of $5 \mathrm{~s}$ for ${ }^{1} \mathrm{H}$ and a typical $\pi / 2$ pulse widths of $4.9 \mu \mathrm{s}$ and a pulse space of $4 \mathrm{~s}$ for ${ }^{2} \mathrm{H}$. The chemical shifts are reported in ppm from tetramethylsilane for ${ }^{1} \mathrm{H}$ and from water for ${ }^{2} \mathrm{H}$.

Fig. 1 shows an example of the 3D plots of static ${ }^{2} \mathrm{H}$ NMR spectra of the system d-heptanol. The $3 \mathrm{D}$ plots show a progressive broadening and a reduction on their intensity as the temperature decreases. The intensity variation exhibits a plateau over the temperatures $240 \mathrm{~K},-33^{\circ} \mathrm{C}$, for $\mathrm{d}$-heptanol. The signal has almost disappeared by the lowest temperature. Similar behaviour was shown for static ${ }^{1} \mathrm{H}$ NMR spectra and the rest of the systems. Each NMR signals were integrate in the frequency range of the liquid one and the normalized values will be analyzed as a function of temperature (Fig. 2 and Fig. 3 ) in the results section.

\section{Results}

Fig. 2 and Fig. 3 display the normalized integrated intensity values for the ${ }^{2} \mathrm{H}$ and ${ }^{1} \mathrm{H}$ NMR signals, corresponding to $\mathrm{d}$-heptanol/h-decane, $\mathrm{d}$-heptanol/h-octane and $\mathrm{d}$-octanol/h-octane binary mixtures adsorbed on graphite. The intensities were determined by numerical integration of the ${ }^{2} \mathrm{H}$ and ${ }^{1} \mathrm{H}$ peaks and normalized to the total number of nominal monolayers. The total amplitude loss observed during the temperature increase due to Curie's law and $Q$ factor effects on excitation and detection have been corrected [13] and [14]. In order to interpret the data from a more quantitative point of view two considerations can be made: (a) the areas under the NMR signals from all the spectra obtained from a given system should be constant, when evaporation is come up, and proportional to the number of the NMR-active species [15] and (b) the strong enhancement of the line-widths at the lower temperatures should be assumed to indicate the onset of the $2 \mathrm{D}$ solid/liquid phase transition [16]. In that case, the NMR resonance line-width is expected to be given approximately by

$\delta u=\alpha_{s}(\delta u)_{s}+\left(1-\alpha_{s}\right)(\delta u)_{1}$

where $(\delta u)_{s}$ and $(\delta v)_{।}$ are the line-widths in the 2D solid monocrystallites and in the liquid, respectively, and where $\alpha_{s}$ and $\left(1-\alpha_{s}\right)$ are the corresponding fractional lifetimes or, equivalently, the fractions of molecules in the solid and liquid phases.

The plots corresponding to Fig. 2 show, from both ${ }^{2} \mathrm{H}$ and ${ }^{1} \mathrm{H}$ signals, the existence of a twostep process. In the first part, at temperatures range from $-55^{\circ}$ to $-33^{\circ} \mathrm{C}$ and from $-33^{\circ}$ to $-13^{\circ} \mathrm{C}$, for d-heptanol and d-octanol (Fig. 2a, c and e), and at temperatures range from $-53^{\circ}$ to $-31^{\circ} \mathrm{C}$ and from $-63^{\circ}$ to $-47^{\circ} \mathrm{C}$, for h-decane and h-octane (Figs. $2 \mathrm{~b}$, $\mathrm{d}$ and f), an increase in the intensity, with a coincident drastic reduction in the line width of the signal is observed. These observations indicate significant mobility of the alcohol and alkane bulk phases. The first step is associated with the bulk melting of both components of the binary mixtures. Above these temperatures there is almost constant intensity observed in both graphs which is attributed to the existence of a remaining 2D solid phase. Finally, at temperatures above $-17^{\circ} \mathrm{C}$ and $11^{\circ} \mathrm{C}$ for $\mathrm{d}$-heptanol and d-octanol (Figs. 3a, $\mathrm{c}$ and e) and above $32{ }^{\circ} \mathrm{C}$ and $-31{ }^{\circ} \mathrm{C}$ for $\mathrm{h}$-decane and h-octane (Figs. $3 \mathrm{~b}, \mathrm{~d}$ and $\mathrm{f}$ ) a further increase of the intensity values is caused by the melting of the $2 \mathrm{D}$ solid phase.

An estimate of the amount of solid which remains just above the bulk melting point can be made, as reported elsewhere [8]. In Fig. 3, the expansions of the second transition region are shown and the results indicate that only a single solid monolayer survives on the graphite surface, as seen from the relative intensities. In this region, the intensity of the ${ }^{2} \mathrm{H}$ and ${ }^{1} \mathrm{H}$ 
signals decrease as the amount of $h$-alkane or d-alcohol increase in the mixture. In the $d$ heptanol/h-decane system, the decrease is more evident in the ${ }^{2} \mathrm{H}$ signal which indicates that there is preferential adsorption of decane. In the d-heptanol/h-octane and d-octanol/h-octane systems, the decrease is more evident in the ${ }^{1} \mathrm{H}$ signal which indicates that the amount of alcohol in the monolayer is higher than the amount of alkane.

The fraction of solid alcohol in the monolayer as a function of bulk composition has been plotted in Fig. 4.

In the $d$-heptanol/h-decane system, there is almost equal adsorption between the alcohol and the alkane at $X_{\text {decane }}=0.20$ and the $\mathrm{h}$-heptanol is essentially completely displaced by decane on the graphite surface at $X_{\text {decane }}=0.30$.

In the $d$-heptanol/h-octane system, there is almost equal adsorption between the alcohol and the alkane at $X_{\text {heptanol }}=0.10$ and the octane is essentially completely displaced by $\mathrm{n}$-heptanol on the graphite surface at a $X_{\text {heptanol }}=0.40$.

In the $\mathrm{d}$-octanol/h-octane system, there is almost equal adsorption between the alcohol and the alkane at a $X_{\text {octanol }}=0.05$ and the octane is essentially completely displaced by $n$-octanol on the graphite surface at a $X_{\text {octanol }}=0.10$.

\section{Discussion}

Fig. 5 shows the plots of the monolayer composition of alcohol as a function of the bulk composition. A different type of behaviour is observed for each combination of alkane and alcohol. The general modelling of sorption isotherms by Giles et al. [17] proposes that 4 main shapes of isotherm are commonly observed for the absorption of liquid onto solid surfaces. The classification of sorption isotherms was based on their initial slopes and curvatures [18]. They distinguished between high affinity, Langmuir, constant partition, and sigmoidal-shaped. Here, we will use this classification to describe data using a qualitative criteria and without mathematical formalism.

The plot of $d$-heptanol/h-decane system shows a sigmoidal-shaped and it can be explained because there is a competitive adsorption of the decane and heptanol in the surface and only at high heptanol concentration is the alcohol adsorption favoured. It is possible that solute-solute attractive forces at the surface may cause cooperative adsorption.

The plot of $d$-heptanol/h-octane system resembles a Langmuir adsorption in which the ratio between the concentration of the compound remaining in bulk and adsorbed on 
the solid decreases when the solute concentration increases, providing a concave curve. It suggests a progressive saturation of the surface.

The plot of the $d$-octanol/h-octane system shows a very high initial slope. This case was distinguished from the others because the compound exhibits a high affinity for the surface.

The preferential absorption of one component over the other has also been analyzed thorough use of an equilibrium coefficient, $K$, based on the equilibrium outlined by Everett [19]

Alkane(bulk)+Alcohol(ads) $\leftrightarrow$ Alkane(ads)+Alcohol(bulk)

where $K=\chi_{\text {alkane }}\left(\right.$ ads). $X_{\text {alcohol }}\left(\right.$ bulk) $/ \chi_{\text {alkane }}\left(\right.$ bulk). $X_{\text {alcohol }}($ ads). When both species are equally adsorbed, $K$ will be unity and preferential adsorption of the alkane will be reflected by a $K$ value greater than unity. Fig. 6 shows the best fits for the three systems of interest here and, only, in the case of the $d$-heptanol/h-octane system, a Langmuir adsorption behaviour, a good fit $\left(R^{2}=0.9993\right)$ was found with a $K_{C 8}=0.076$ that suggests a preferential adsorption of heptanol over octane.

The relative size ratio is a measure of mismatch in molecule size compared to the overall sizes of the molecules calculated as the difference in alkyl chain lengths of the two components divided by the average chain length, as follows [20]:

$$
R S=2 \frac{|m-n|}{m+n}
$$

The molar fraction of alcohol for a $95 \%$ of monolayer composition as a function of the relative size ration (no shown) reveals that a relative size ratio of 0.133 gives approximately equal preferential adsorption between an alcohol and an alkane. The $\mathrm{OH}$ group of alcohol allows them to form strong hydrogen bonds which increase the length of the alcohol molecules and leads to a stronger adsorption. However, the data presented above seems to indicate that the length of the molecule is not the only factor because the equivalence in adsorption is not between an alcohol of length $m$ and the alkane with a length of $n=2 m$ but only with an alkane $15 \%$ longer.

\section{Conclusions}

It has been demonstrated that the combine use of non-deuterated and deuterated materials allows analyzing the different behaviour of mixed alkane/alcohol, with similar ${ }^{1} \mathrm{H}$ chemical shift values.

There is a preferential adsorption of the components: it is clear that longer molecule is preferentially adsorbed, as observed in the alkane/alkane and alcohol/alcohol binary mixture (decane vs. heptanol). If both components are of similar length, the alcohol is preferentially adsorbed (heptanol vs. octane and octanol vs. octane). Finally, a linear relation between the relative size ratio and the amount of alcohol for the monolayer covering is observed. 


\section{Acknowledgments}

We gratefully acknowledge financial support from Ministerio del Medio Ambiente y Medio Rural y Marino Project no. 300/PC08/3-01.1 and DGICYT Projects no. CTQ2010-14874.

\section{References}

[1]

L. Messe, A. Perdigon, S.M. Clarke, A. Inaba, T. Arnold

Langmuir, 21 (2005), pp. 5085-5093

[2]

E.P. Gilbert, P.A. Reynolds, P. Thiyagarajan, D.G. Wozniak, J.W. White

Phys. Chem. Chem. Phys., 1 (1999), pp. 2715-2724

[3]

L. Messe, S.,.M. Clarke, A. Inaba, C.C. Dong, R.K. Thomas, M.A. Castro, M.D. Alba

Langmuir, 18 (2002), pp. 9429-9433

[4]

G.H. Findenegg, C. Koch, M. Liphard, Adsorption from Solution, in: Proceedings of the Conference in Honour of D. H. Everett, Bristol, Sept. 8-10, 1982.

[5]

S.M. Clarke, L. Messe, J. Adams, A. Inaba, T. Arnold, R.K. Thomas

Chem. Phys. Lett., 373 (2003), pp. 480-485

[6]

M.A. Castro, S.M. Clarke, A. Inaba, C.C. Dong, R.K. Thomas

J. Phys. Chem. B, 102 (1998), pp. 777-781

[7]

M.A. Castro, S.M. Clarke, A. Inaba, R.K. Thomas

Physica B, 241-243 (1998), pp. 1086-1088

[8]

M.D. Alba, M.A. Castro, S.M. Clarke, A.C. Perdigón

Solid State Nucl. Magn. Reson., 23 (2003), pp. 174-181 
[9]

M.D. Alba, M.A. Castro, S.M. Clarke, S. Medina, L. Messe, C. Millan, M.M. Orta, A.C. Perdigón

J. Phys. Chem. C, 113 (2009), pp. 3176-3180

[10]

G. Neue, B. Boddenberg

Mol. Phys., 68 (1989), pp. 771-790

[11]

A.J. Groszek

Proc. Roy. Soc. Lond., A314 (1970), p. 473 \&

[12]

K. Morishige, T.J. Kato

Chem. Phys, 111 (1999), pp. 7095-7102

[13]

W.G. Clark

Rev. Sci. Instrum, 35 (1964), p. 316 \&

[14]

C. Gerardin, M. Haouas, C. Lorentz, F. Taulelle

Magn. Reson. Chem., 38 (2000), pp. 429-435

[15]

R.K. Harris

Nuclear Magnetic Resonance Spectroscopy

Longman, Scientific \& Technical, New York (1986)

[16]

V. Grundke, B. Boddenberg

Mol. Phys., 79 (1993), pp. 1215-1226

[17]

C.H. Giles, D. Smith, A. Huston 
J. Collis. Interface Sci., 47 (1974), pp. 755-765

[18]

C. Hinz

Geoderma, 99 (2001), pp. 225-243

[19]

D.H. Everett

R.H. Ottewell, C.H. Rochester, A.L. Smith (Eds.), Adsorption from Solution, Academic Press, London (1982)

[20]

T. Arnold, Ph.D. Thesis, Oxford, 2001. 


\section{Figure captions}

Figure 1. 3D plots of static ${ }^{2} \mathrm{H}$ NMR spectra of the system d-heptanol.

Figure 2. Static ${ }^{2} H$ NMR (left) and ${ }^{1} H$ NMR (right) data from $(a, b) d$-heptanol/h-decane mixtures, (c,d) d-heptanol/h-octane mixtures and $(e, f) d$-octanol/h-octane mixtures adsorbed on graphite as a function of temperature for a range of solution compositions.

Figure 3. Static ${ }^{2} \mathrm{H} N M R$ (left) and ${ }^{1} \mathrm{H}$ NMR (righ) data of monolayer melting point region from $(a, b) d$-heptanol/h-decane mixtures, (c,d) d-heptanol/h-octane mixtures and (e,f) d-octanol/hoctane mixtures adsorbed on graphite as a function of temperature for a range of solution compositions.

Figure 4. Surface composition of: (a,b) d-heptanol/h-decane mixtures, (c,d) d-heptanol/hoctane mixtures and $(e, f) d$-octanol/h-octane mixtures adsorbed on graphite as a function of bulk composition.

Figure 5. Experimental determined monolayer composition of alcohol as a function of the bulk composition.

Figure 6. Surface composition of alcohol as a function of the bulk composition: (a) $d$ heptanol/h-decane mixtures, (b) d-heptanol/h-octane mixtures and (c) d-octanol/h-octane mixtures. Dots=experimental data, and, solid line=fit data. 
Figure 1

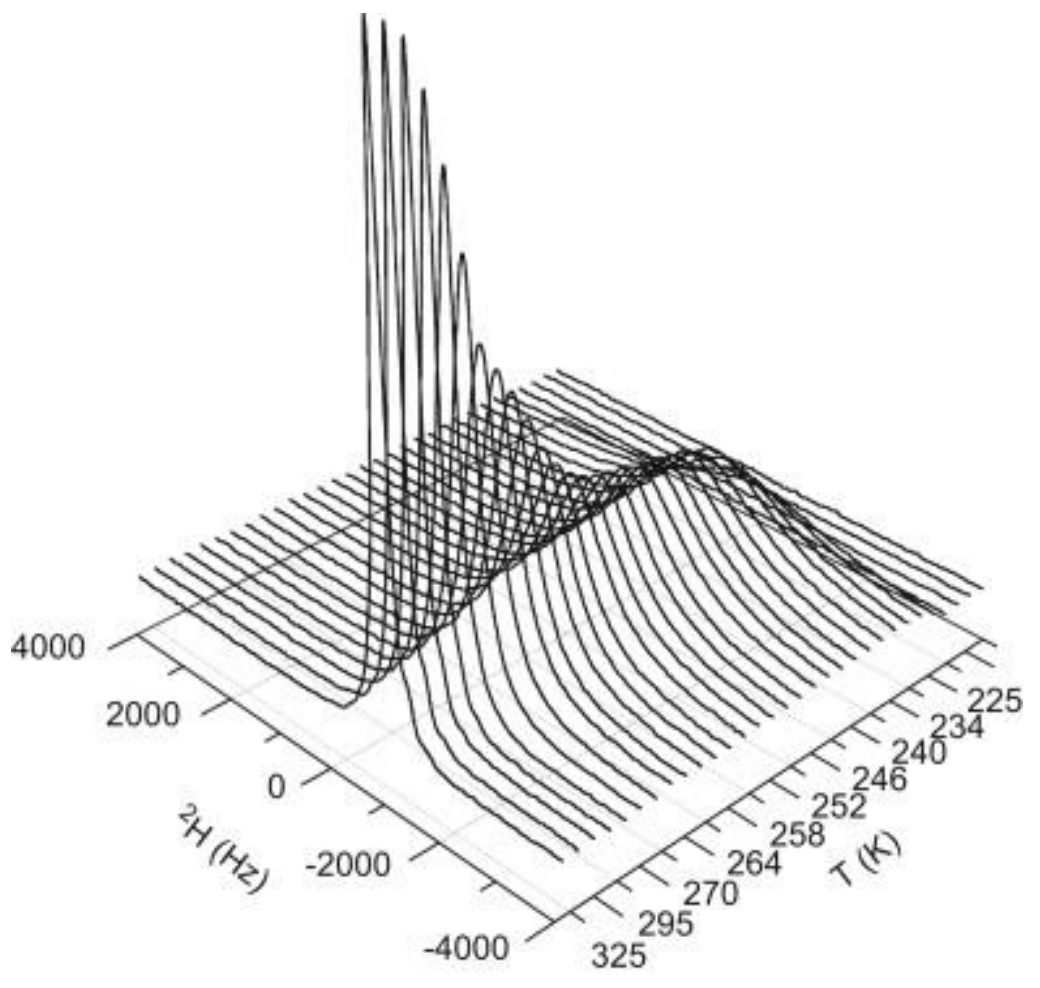


Figure 2

a

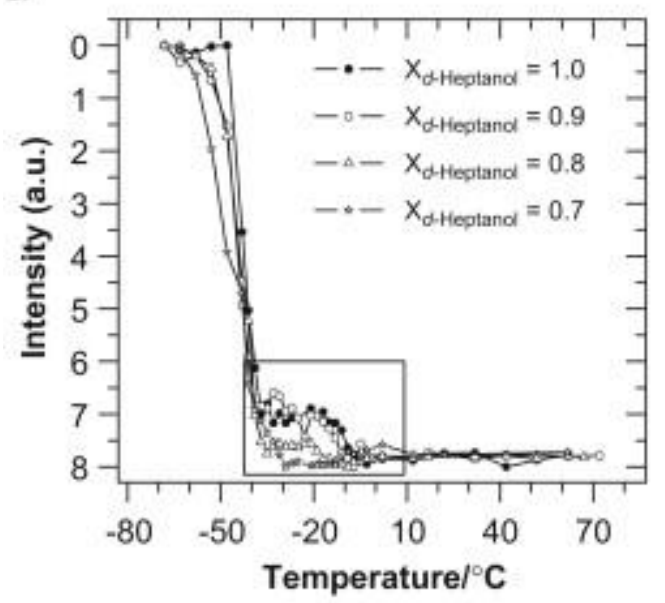

C

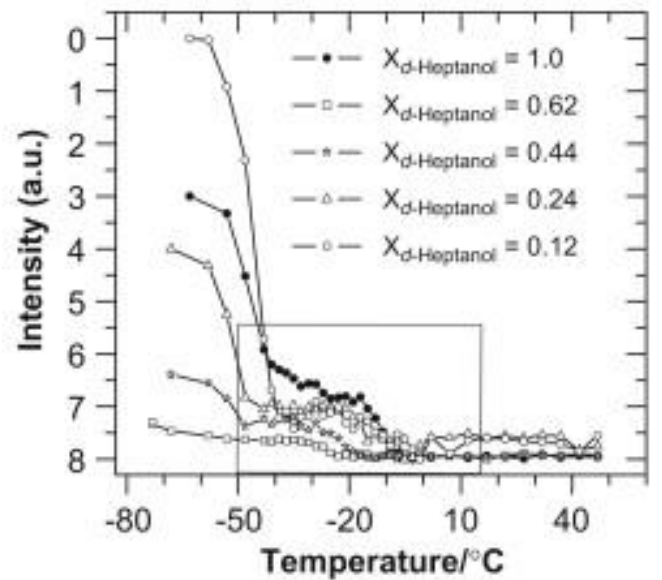

e

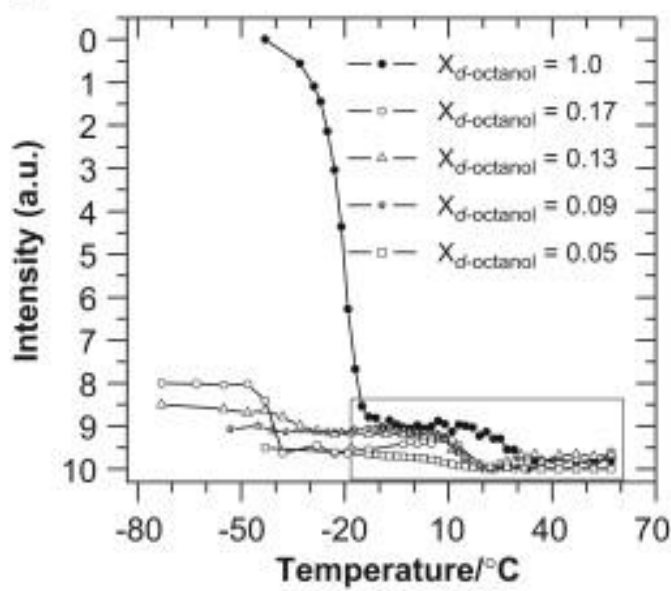

b

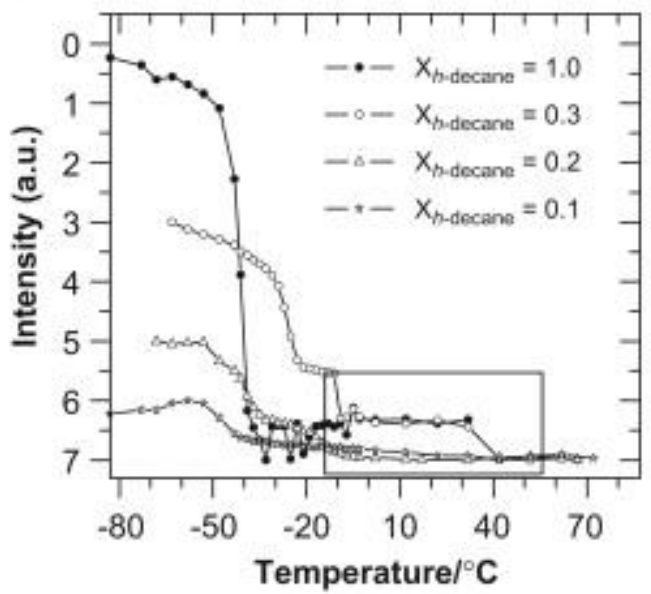

d

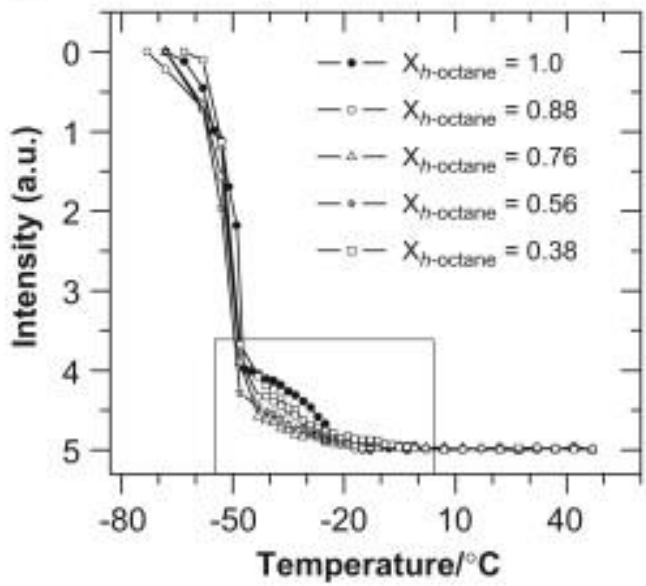

f

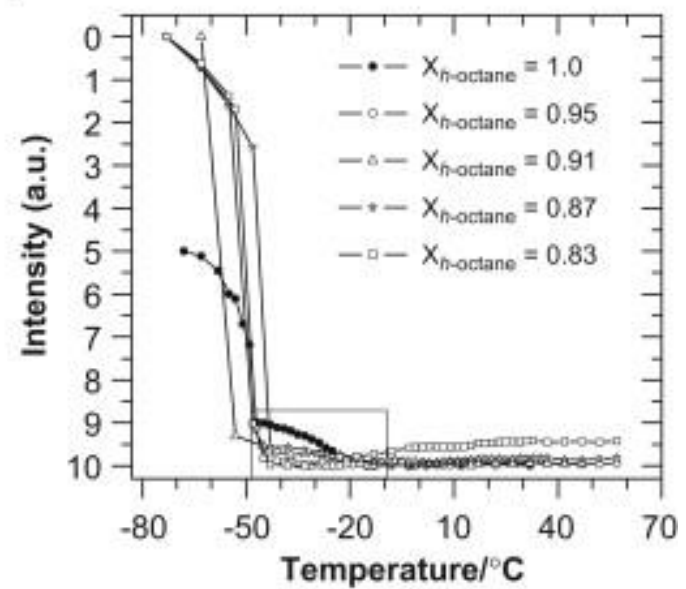




\section{Figure 3}

a

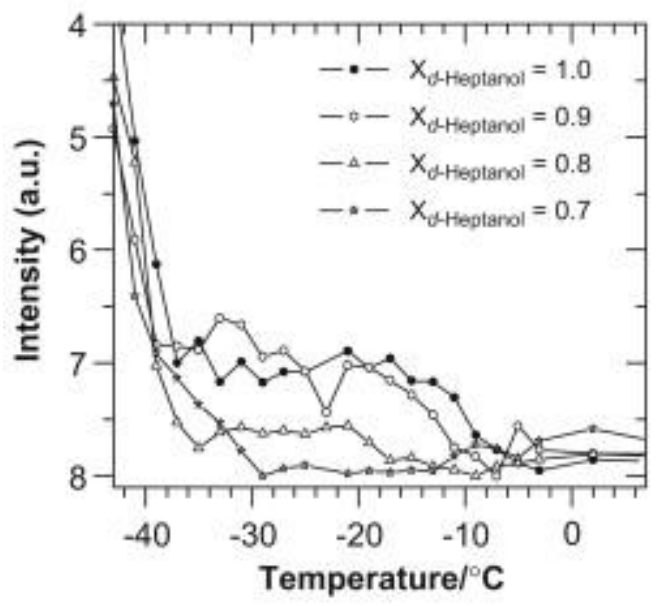

C

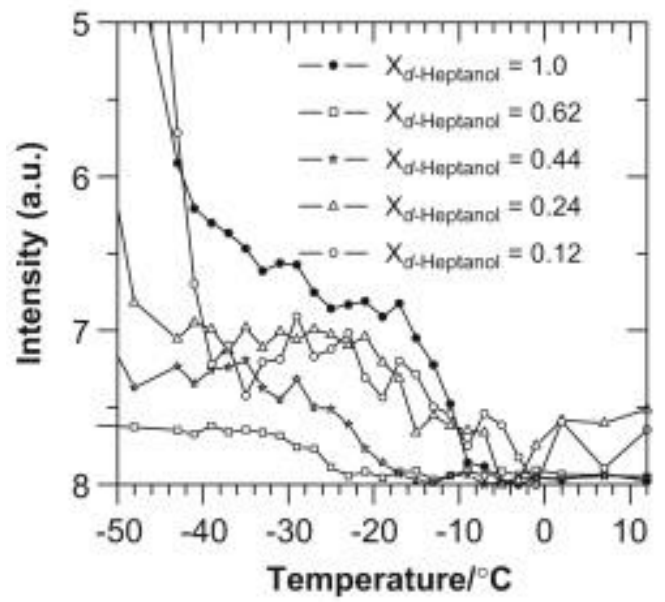

e

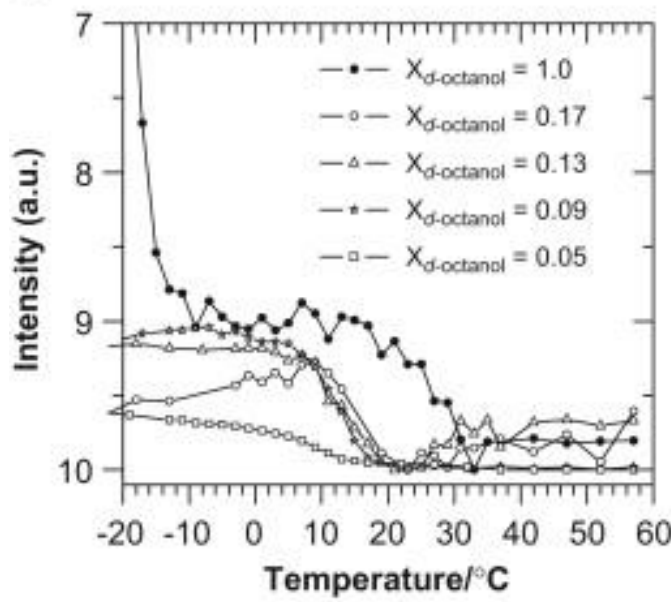

b

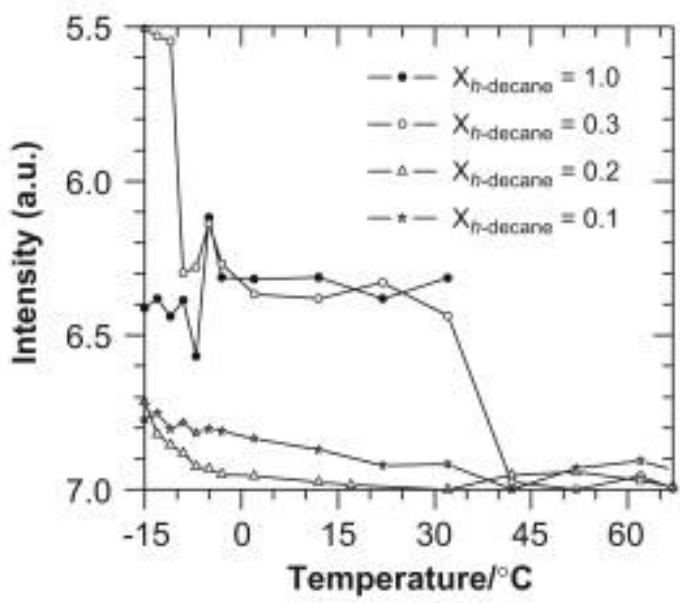

d

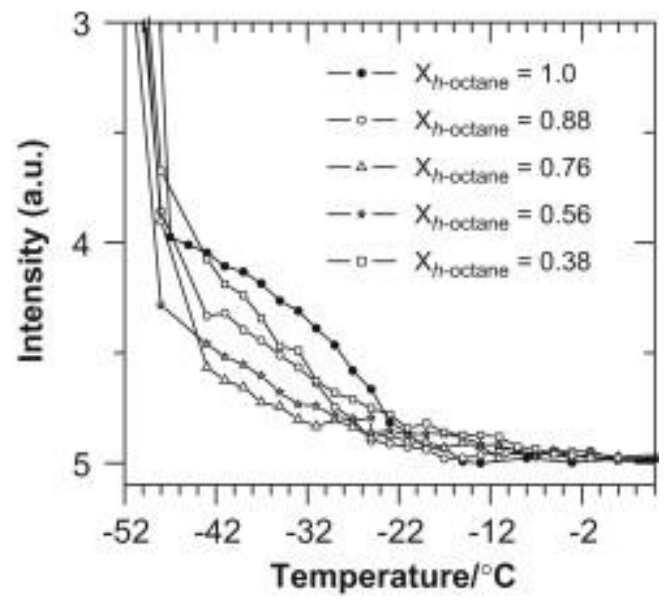

f

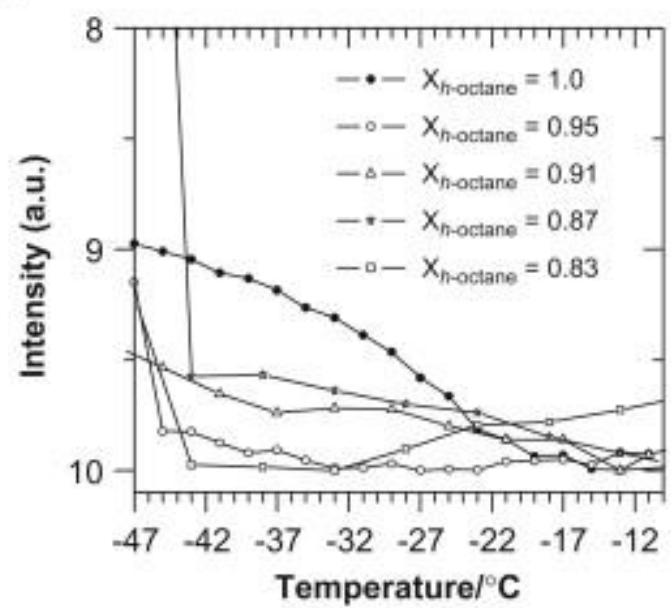


Figure 4
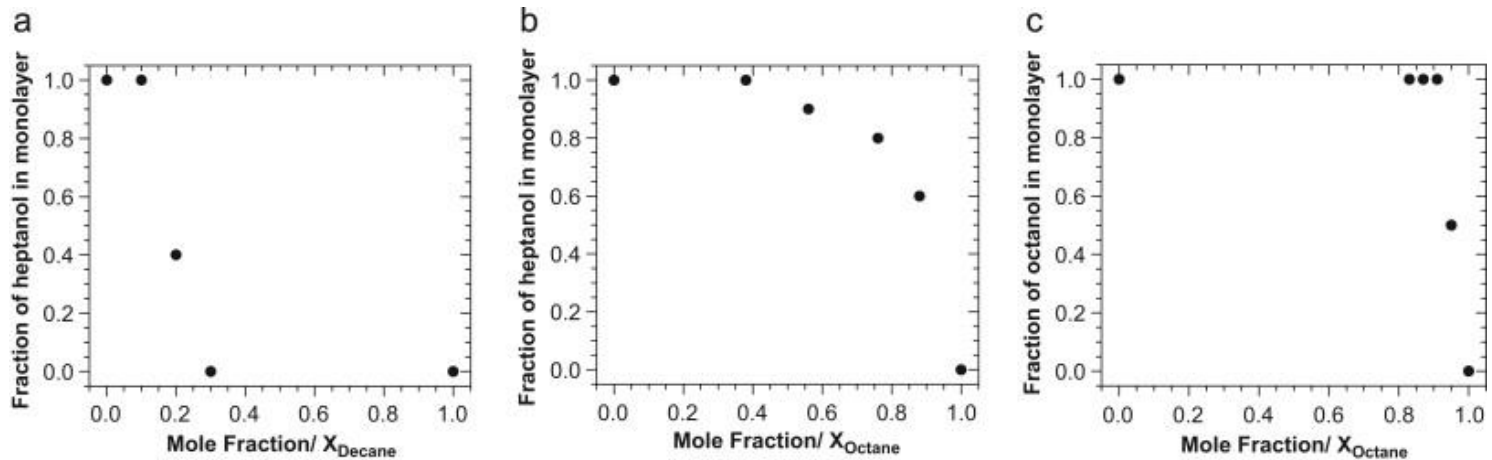
Figure 5.

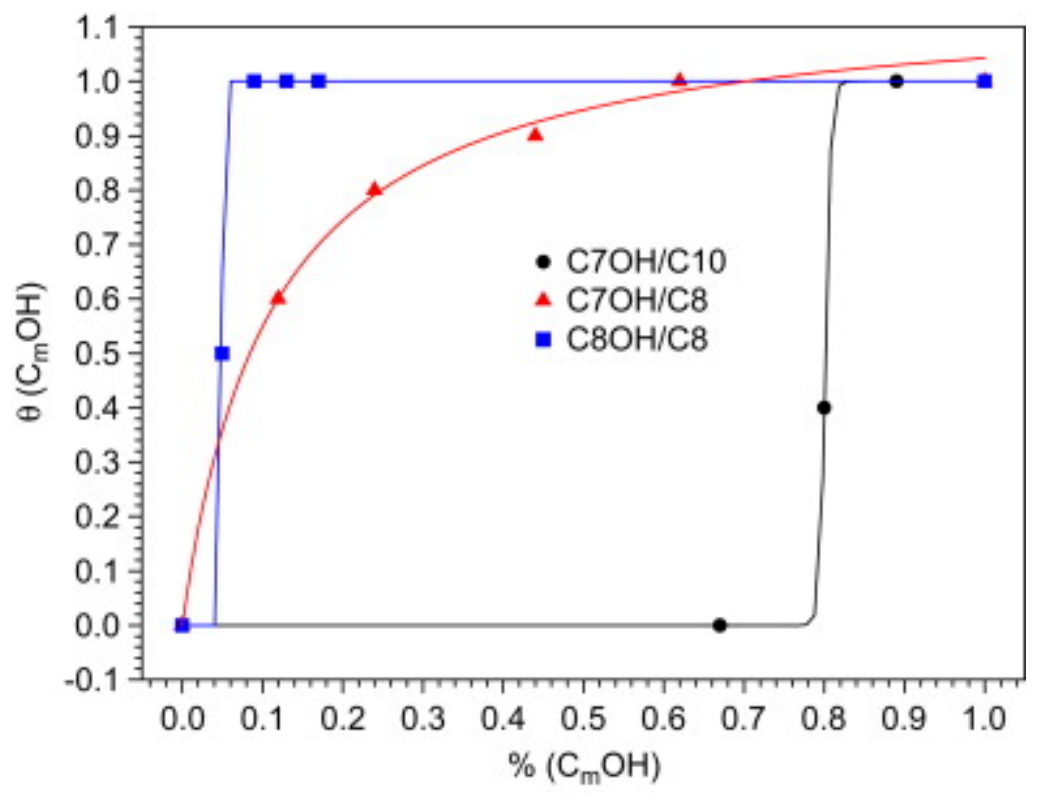


Figure 6
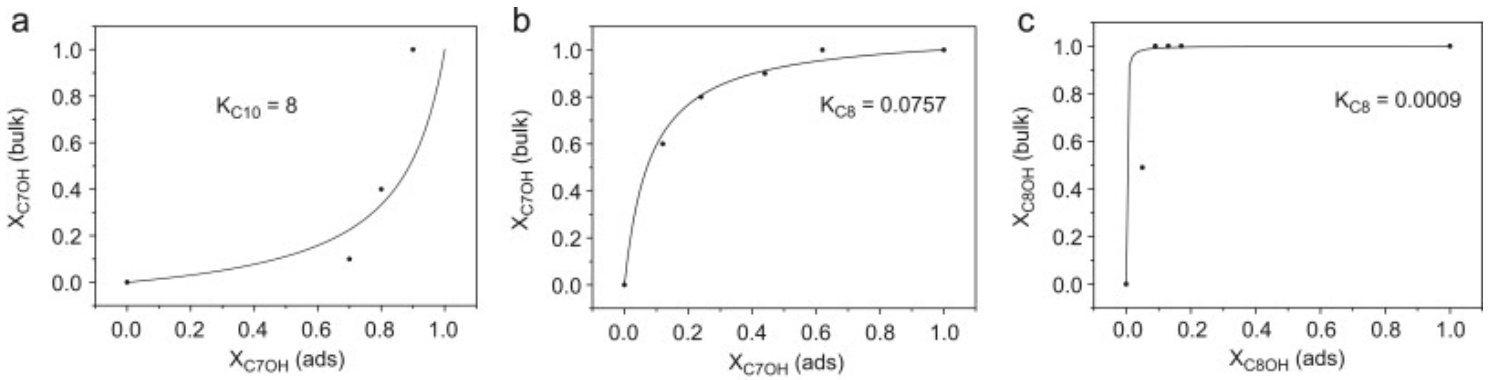